\title{
ANOMALOUS SKIN EFFECT IN CYLINDRICAL SAMPLES
}

\author{
H. P. VAN DE BRAAK and L. J. M. VAN DE KLUNDERT \\ Technische Hogeschool Twente, Enschede, The Netherlands
}

Received 14 August 1974

\section{Synopsis}

A description is given of the anomalous skin effect in a cylinder placed in an a.c. magnetic field parallel to the cylinder axis. The necessary nonlocal relation between current density and electric field inside the sample is established with the aid of Boltzmann's transport equation. Results are presented in terms of the current density in the sample and its magnetic susceptibility.

1. Introduction. It is a well-known fact that in a metal, placed in an electromagnetic field, the internal fields are damped out rapidly due to the skin effect. The usual description of this phenomenon is based on Maxwell's equations and Ohm's law, leading to the introduction of the skin depth, i.e., the distance over which the field penetrates in the metal. Pippard ${ }^{1}$ ) was the first to point out that the implicit assumption that the field may be considered as spatially constant for the purpose of calculating the current at a point, cannot be generally valid. The theory will certainly break down when the field varies appreciably over a distance of the order of the mean free path of the conduction electrons. This effect, which is observed at low temperatures where the mean free path is large, is known as the anomalous skin effect and is characterized by a further decrease of the bulk conductivity of the metal.

This has been discussed at length by Reuter and Sondheimer ${ }^{2}$ ) and several other authors ${ }^{3,4}$ ) on the basis of a more general relation between current and electric field, in which the expression for the local current density involves a definite integral over the values of the field at all points in the metal. Such a relation can be derived from Boltzmann's transport equation for the conduction electrons.

The case of a cylindrical sample was first considered by Dingle ${ }^{5}$ ) for a wire in a homogeneous electric field in the direction of the wire. It is the purpose of the present paper to extend the theory to a case which is of particular interest from an experimental point of view, namely a cylinder 
placed in a solenoid $\left.{ }^{6}\right)$. The external magnetic field may be considered axial and homogeneous and gives rise to circular electric fields and currents inside the sample. For reasons of convenience we suppose the cylinder to be infinitely long.

The relevant measurable quantity in this situation is the bulk magnetic permeability rather than the bulk resistivity, where the permeability is defined as the ratio between the total magnetic induction in the sample and the value of the external induction multiplied by the sample volume. In section 2 of this paper we shall give a phenomenological description based on Maxwell's equations and a general form of the relation between the current density $j$ and the electric field $\boldsymbol{E}$. It is assumed that the material is homogeneous and isotropic. We find an expression for the susceptibility in terms of $j$ and an integral equation for $j$.

The kernel of this equation depends on the specific form of the relation between $j$ and $E$. This relation will be derived in section 3, where we start with solving Boltzmann's equation for a system with cylindrical geometry. We restrict ourselves to the case that the reflection of the electrons at the surface of the sample is completely diffuse ${ }^{7}$ ) and the Fermi surface spherical.

2. Phenomenological description. We consider an infinitely long cylinder, placed in an a.c. magnetic field parallel to the cylinder axis. For reasons of symmetry the magnetic field inside the sample has only an axial component, whereas for the electric field the axial component vanishes.

Putting $B(t)=B \exp (-\mathrm{i} \omega t)$ and $E(t)=E \exp (-\mathrm{i} \omega t)$ we can write Maxwell's equation in the following form

$$
\begin{aligned}
& \mathrm{i} \omega B_{z}=\frac{\mathrm{d} E_{\theta}}{\mathrm{d} r}+\frac{E_{\theta}}{r} ; \quad-\frac{\mathrm{d} H_{z}}{\mathrm{~d} r}=j_{\theta}-\mathrm{i} \omega D_{\theta}, \\
& i_{r}=\mathrm{i} \omega D_{r} ; \quad \frac{\mathrm{d} D_{r}}{\mathrm{~d} r}+\frac{D_{r}}{r}=\rho .
\end{aligned}
$$

For all practical purposes one may neglect the displacement current in (2.1).

In the case of linear response in an isotropic medium we expect a relation between the current and the electric field, which has the following general form

$$
j(r)=\sigma_{0} \int_{0}^{a} F(r, y) E(y) \mathrm{d} y,
$$


where $\sigma_{0}$ is the d.c. conductivity and $a$ the radius of the sample. If we assume further, that the medium is characterized by the constitutive relations $B=\mu_{0} H$ and $D=\epsilon_{0} E$, it is obvious from eq. (2.2) that the only consistent description of the system implies $j_{r}=F_{r}=0$. As a consequence the space charge $\rho$ must also be neglected.

So the relevant equations describing the fields and current in the system are

$$
\mathrm{i} \omega B=\frac{\mathrm{d} E}{\mathrm{~d} r}+\frac{E}{r} ; \quad \frac{\mathrm{d} B}{\mathrm{~d} r}=-\mu_{0} j,
$$

where we have dropped the indices since no confusion can arise.

Now we can solve eq. (2.3) and (2.4) for any of the three variables. For reasons of computational convenience we choose a solution in terms of $j(r)$. (For a solution in terms of $E$, which can be used to check on the results, see the end of this section.)

By integraton of (2.4) we get

$$
\begin{aligned}
& E(r)=\frac{1}{2} \mathrm{i} \omega r B(r)+\frac{1}{2} \mathrm{i} \omega \mu_{0} \int_{0}^{r} x^{2} j(x) \mathrm{d} x, \\
& B(r)=B_{\mathrm{a}}+\mu_{0} \int_{r}^{a} j(x) \mathrm{d} x .
\end{aligned}
$$

Here we have used the boundary conditions $E(0)=0$ and $B(a)=B_{a}$, the amplitude of the applied external field. The two equations above can be combined with (2.3) by simple substitution, and we are led to the following integral equation

$$
\begin{aligned}
j(r) & =\frac{1}{2} \mathrm{i} \omega \sigma_{0} B_{a} \int_{0}^{a} y F(r, y) \mathrm{d} y \\
& +\frac{1}{2} \mathrm{i} \omega \mu_{0} \sigma_{0} \int_{0}^{a} j(x)\left(\int_{0}^{x} y F(r, y) \mathrm{d} y+\int_{x}^{a}\left(x^{2} / y\right) F(r, y) \mathrm{d} y\right) \mathrm{d} x .
\end{aligned}
$$

Since we are interested in the magnetic susceptibility, which is independent of the magnitude of the applied field, it is convenient to dispose of $B_{u}$ by considering the quantity

$$
J(r)=\mu_{0} j(r) / B_{a} .
$$

Substitition of (2.6) into (2.5) yields

$$
\begin{aligned}
J(r) & =\frac{1}{2} \kappa^{2} \int_{0}^{a} y F(r, y) \mathrm{d} y \\
& +\frac{1}{2} \kappa^{2} \int_{0}^{a} J(x)\left(\int_{0}^{x} y F(r, y) \mathrm{d} y+\int_{x}^{a}\left(x^{2} / y\right) F(r, y) \mathrm{d} y\right),
\end{aligned}
$$


where $\kappa^{2}=i \omega \mu_{0} \sigma_{0}$. This quantity is related to the normal skin depth in the usual way: $\operatorname{Im} \kappa=\delta^{\sim 1}$.

Further simplification is obtained by defining an integral kernel

$$
G(r, x)=\int_{0}^{x} y F(r, y) \mathrm{d} y+\int_{x}^{a}\left(x^{2} / y\right) F(r, y) \mathrm{d} y .
$$

So we have

$$
J(r)=\frac{1}{2} \kappa^{2} G(r, a)+\frac{1}{2} \kappa^{2} \int_{0}^{a} G(r, x) J(x) \mathrm{d} x .
$$

We define the magnetic susceptibility of the sample as follows:

$$
\chi=-1+\frac{\int B(r) \mathrm{d} O}{B_{\mathrm{a}} \int \mathrm{d} O}
$$

where $\mathrm{d} O$ is an element of a cross section of the sample. With the aid of the second equation of (2.4) and integration by parts one readily finds

$$
\chi=\left(1 / a^{2}\right) \int_{0}^{a} r^{2} J(r) \mathrm{d} r .
$$

From (2.8) and (2.9) the susceptibility can be calculated once the function $F(r, y)$, relating current to electric field, is known. We shall deal with this problem in detail in section 3 of this paper.

In the case of the normal skin effect, however, it is not necessary to calculate $\chi$ in the elaborate way sketched above. In fact, eq. (2.3) reduces to Ohm's law and from (2.4) we then get

$$
\frac{\mathrm{d}^{2} J}{\mathrm{~d} r^{2}}+\frac{1}{r} \frac{\mathrm{d} J}{\mathrm{~d} r}+\left(\kappa^{2}-\frac{1}{r^{2}}\right) J=0
$$

In this case the susceptibility can be expressed in terms of Bessel functions

$$
\chi=\mathscr{I}_{2}(\kappa a) / \mathscr{g}_{0}(\kappa a)
$$

To conclude this section we shall indicate how one can solve for the susceptibility in terms of $E$ rather than $j$. By elimination of $B$ and $j$ from (2.3) and (2.4) one can derive the following integral equation

$$
E(r)=C r-\left(\kappa^{2} / 2 r\right) \int_{0}^{a} \mathrm{~d} y E(y) \int_{0}^{r} \mathrm{~d} x\left(r^{2}-x^{2}\right) F(x, y),
$$


where $C$ is a constant connected with the amplitude of the applied field. As mentioned above this constant will have no influence of the value of $\chi$. The susceptibility can be expressed in terms of $E$ in the following way:

$$
\chi=\left[E(a)-a E^{\prime}(a)\right] /\left[E(a)+a E^{\prime}(a)\right] .
$$

3. Transport theory. In this section we shall establish a relation between $j$ and $E$ based on Boltzmann's transport equation. For cylindrical samples this problem was first attacked by Dingle ${ }^{5}$ ) for the case of an axial, homogeneous electric field. As we pointed out in the previous section, we have to deal with a field with only a $\theta$ component depending on $r$. Our treatment differs from Dingle's also in this respect that we prefer to use cylindrical coordinates $r, \theta$ and $z$ and their canonical momenta rather than cartesian coordinates and velocities. In this way centrifugal effects can be handled more easily. So we put $x=r \cos \theta, y=r \sin \theta$. The canonical momenta for the system in equilibrium are defined as follows:

$$
p_{r}=m \dot{r} ; \quad p_{\theta}=m r^{2} \dot{\theta} ; \quad p_{z}=m \dot{z} .
$$

The corresponding equations of motion in the presence of the field are:

$$
\dot{p}_{r}=p_{\theta}^{2} / m r^{3}, \quad \dot{p}_{\theta}=-|\mathrm{e}| r E, \quad \dot{p}_{z}=0,
$$

where $|e|$ is the elementary charge. The effect of the magnetic field on the motion of the electrons is neglected.

The distribution function in phase space $f(r, p, t)$ will depend on the coordinate $r$ and all three components of $p$. So we can write Boltzmann's equation in the following way

$$
\frac{\partial f}{\partial t}+\dot{r} \frac{\partial f}{\partial r}+\dot{p}_{r} \frac{\partial f}{\partial p_{r}}+\dot{p}_{\theta} \frac{\partial f}{\partial p_{\theta}}+\dot{p}_{z} \frac{\partial f}{\partial p_{z}}=\left(\frac{\partial f}{\partial t}\right)_{\text {coll }}
$$

We put $f=f_{0}+f_{1}$ where $f_{0}$ is the equilibrium distribution which depends only on the kinetic energy $\epsilon$. If we approximate the collision term by introducing a relaxation time and linearize the equation with respect to $E$, we find

$$
\left(\frac{1}{\tau}-\mathrm{i} \omega\right) f_{1}+\left(\frac{p_{r}}{m} \frac{\partial}{\partial r}+\frac{p_{\theta}^{2}}{m r^{3}} \frac{\partial}{\partial p_{r}}\right) f_{1}=\frac{|e|}{m} \frac{\partial f_{0}}{\partial \epsilon} \frac{E p_{\theta}}{r} .
$$

For the frequencies we are interested in we may neglect i $\omega$ compared to $\tau^{-1}$. In order to make our calculations consistent with the description 
given in section 2, we must require that the solution of (3.2) be such that it gives no contribution for the radial current and the charge density. So we have to apply the subsidiary conditions

$$
\int f_{1}(r, v) v_{r} \mathrm{~d}^{3} v=0, \quad \int f_{1}(r, v) \mathrm{d}^{3} v=0 .
$$

The formal solution of eq. (3.2) can be obtained by means of the following transformation.

$$
\xi=p_{r}^{2}+p_{\theta}^{2} / r^{2}, \quad \eta=r, \zeta=p_{\theta},
$$

with the inverse transformation $p_{r}= \pm\left(\xi-\zeta^{2} / \eta^{2}\right)^{\frac{1}{2}}$. The square root is taken positive. We find

$$
\frac{\partial f_{1}}{\partial \eta}+\frac{m \eta}{\tau\left(\xi \eta^{2}-\zeta^{2}\right)^{\frac{1}{2}}} f_{1}= \pm 2|e| m \frac{\partial f_{0}}{\partial \xi} \frac{\zeta E(\eta)}{\left(\xi \eta^{2}-\zeta^{2}\right)^{\frac{1}{2}}}
$$

the upper sign referring to positive $p_{r}$ values, the lower sign to the negative ones. We call the two corresponding branches of the solution $f_{1}^{+}$and $f_{1}^{-}$ respectively. The solution is

$$
\begin{aligned}
f_{1}^{ \pm}= & \mathrm{e}^{\mp(m / \tau \xi)\left(\xi \eta^{2}-\zeta^{2}\right)^{\frac{1}{2}}} \\
& \times\left( \pm 2|e| m \frac{\partial f_{0}}{\partial \xi} \int^{n} \mathrm{e}^{ \pm(m / \tau \xi)\left(\xi y^{2}-\zeta^{2}\right)^{\frac{1}{2}}} \frac{\zeta E(y)}{\left(\xi y^{2}-\zeta^{2}\right)^{\frac{1}{2}}} \mathrm{~d} y+\psi_{ \pm}(\xi, \zeta)\right) .
\end{aligned}
$$

$\psi_{ \pm}$are arbitrary functions of $\zeta$ and $\xi$, but independent of $\eta$.

Expressing the solution in terms of the original variables and introducing the velocities as variables rather than the momenta according to $p_{r}=m v_{r}$, $p_{\theta}=m r v_{\theta}$, we finally obtain

$$
f_{1}^{ \pm}=\mathrm{e}^{-\alpha r v_{r}}\left( \pm|e| \frac{\partial f_{0}}{\partial \epsilon} \int_{r \pm}^{r} \mathrm{e}^{ \pm \alpha \beta} r v_{\theta} E(y) \beta^{-1} \mathrm{~d} y+\psi_{ \pm}\right),
$$

with

$$
\alpha^{-1}=\tau\left(v_{r}^{2}+\tau_{\theta}^{2}\right), \beta=\left[\left(v_{r}^{2}+v_{\theta}^{2}\right) y^{2}-r^{2} v_{\theta}^{2}\right]^{\frac{1}{2}} .
$$

The arbitrary functions $\psi_{ \pm}$and $r_{ \pm}$depend on the combinations $v_{r}^{2}+v_{\theta}^{2}$, and $r v_{\theta}$. They must be determined by requirements of regularity and 
boundary conditions. For real $\beta$ we must have

$$
y \geqslant r\left|v_{\theta}\right|\left(v_{r}^{2}+v_{\theta}^{2}\right)^{-\frac{1}{2}}
$$

Further we require the exponential functions in (3.5) to remain finite in the limit $\alpha \rightarrow \infty$. This yields the condition $-r v_{r} \pm \beta \leqslant 0$, which condition can be met by taking

$$
y \leqslant r \text { for } v_{r}>0, \quad y \geqslant r \text { for } v_{r}<0 .
$$

Both conditions (3.6) and (3.7) are fulfilled if we put $r_{+}=r\left|v_{\theta}\right|\left(v_{r}^{2}+v_{\theta}^{2}\right)^{-\frac{1}{2}}$, $r^{-}=a$, where $a$ is the radius of the sample. So we have

$$
\begin{aligned}
& f_{1}^{+}=\mathrm{e}^{-\alpha r v_{r}}\left(|\mathcal{e}| \frac{\partial f_{0}}{\partial \epsilon} \int_{r+}^{r} \mathrm{e}^{\alpha \beta} r v_{\theta} E(y) \beta^{-1} \mathrm{~d} y+\psi_{+}\right), \\
& f_{1}^{-}=\mathrm{e}^{-\alpha r v_{r}}\left(|e| \frac{\partial f_{0}}{\partial \epsilon} \int_{r}^{a} \mathrm{a}^{-\alpha \beta} r v_{\theta} E(y) \beta^{-1} \mathrm{~d} y+\psi_{-}\right) .
\end{aligned}
$$

The next requirement is continuity of $f_{1}$ for $v_{r}=0$. Here the integral part of $f_{1}^{+}$vanishes since $r_{+}\left(v_{r}=0\right)=r$, so continuity is obtained if we take

$$
\psi_{+}-\psi_{-}=|e| \frac{\partial f_{0}}{\partial \epsilon} \int_{r+}^{r} \mathrm{e}^{-\alpha \beta} r v_{\theta} E(y) \beta^{-1} \mathrm{~d} y .
$$

One could, of course, add any function $h\left(v_{r}^{2}+v_{\theta}^{2}, r v_{\theta}\right)$ to the right-hand side of (3.9), provided that $h=0$ for $v_{r}=0$. However, it is easy to prove that $h$ must vanish identically.

Finally one has the boundary condition at $r=a$ which specifies the nature of the scattering of the electrons at the surface ${ }^{7}$ ). We assume that the scattering is completely diffuse. This means that the distribution of the electrons leaving the surface will be random with respect to the velocities. So we have $f_{1}^{-}(r=a)=$ constant $C$, and hence $\psi_{-.}(r=a)=C$. It is again easy to prove that $\psi_{-}$will then be identically constant. This constant may be determined from either of the two equations (3.3). For this purpose, and also for the calculation of current densities, we must evaluate integrals in $v$ space. Following Dingle ${ }^{5}$ ) we introduce a set of spherical coordinates for the velocity at every point $(r, \theta, z)$ :

$v_{r}=v \sin \theta \cos \phi, v_{\theta}=v \sin \theta \sin \phi, v_{z}=v \cos \theta$, with $0 \leqslant \theta \leqslant \pi$, and $-\frac{1}{2} \pi \leqslant \phi \leqslant \frac{3}{2} \pi$.

Expressed in these coordinates (3.8) and (3.9) become 
$f_{1}^{+}=\mathrm{e}^{-r \cos \phi / \tau v \sin \theta}$

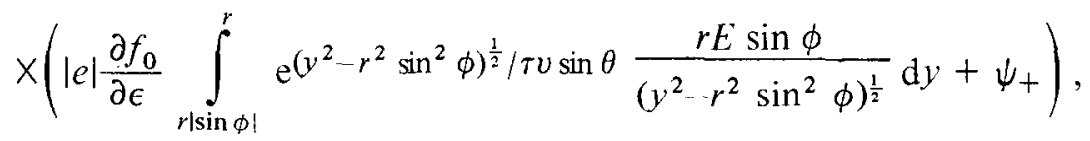

$f_{1}^{-}=\mathrm{e}^{-r \cos \phi / \tau v \sin \theta}$

$$
\times\left(|e| \frac{\partial f_{0}}{\partial \epsilon} \int_{r}^{a} \mathrm{e}^{-\left(y^{2}-r^{2} \sin ^{2} \phi\right)^{\frac{1}{2}} / \tau v \sin \theta} \frac{r E \sin \phi}{\left(y^{2}-r^{2} \sin ^{2} \phi\right)^{\frac{1}{2}}} \mathrm{~d} y+C\right),
$$

$\psi^{+}=\mathrm{e}^{-r \cos \phi / \tau v \sin \theta}$

$$
\times\left(|e| \frac{\partial f_{0}}{\partial \epsilon} \int_{r|\sin \phi|}^{a} \mathrm{e}^{-\left(y^{2}-r^{2} \sin ^{2} \phi\right)^{\frac{1}{2}} / \tau v \sin \theta} \frac{r E \sin \phi}{\left(y^{2}-r^{2} \sin ^{2} \phi\right)^{\frac{1}{2}}} \mathrm{~d} y+C\right)
$$

Now we apply condition (3.3), which states that the space charge density must vanish. Assuming a spherical Fermi surface we have

$$
\int_{0}^{v} \mathrm{~d} v \int_{0}^{\pi} \mathrm{d} \theta\left(\int_{-\frac{1}{2} \pi}^{\frac{1}{2} \pi} \mathrm{d} \phi v^{2} \sin \theta f_{1}^{+}+\int_{\frac{1}{2} \pi}^{\frac{3}{2} \pi} \mathrm{d} \phi v^{2} \sin \theta f_{1}^{-}\right)=0 .
$$

Apart from the terms with the constant $C$ both $f_{1}^{+}$and $f_{1}^{-}$are odd functions of $\phi$, so the only contribution to the integrals comes from the terms with $C$. The latter are even in $\phi$, so (3.10) leads to

$$
C=0
$$

It can be verified that this choice also ensures that $j_{r}=0$. We are now ready to calculate the $\theta$ component of the current

$$
\begin{aligned}
j= & -\left(|e|^{2} m^{3} / 4 \pi^{3} \hbar^{3}\right) \int_{0}^{v} \mathrm{~F} \mathrm{~d} v \int_{0}^{\pi} \mathrm{d} \theta \\
& \times\left(\int_{-\frac{1}{2} \pi}^{\frac{1}{2} \pi} \mathrm{d} \phi v^{3} \sin ^{2} \theta \sin \phi f_{1}^{+}+\int_{\frac{1}{2} \pi}^{\frac{3}{2} \pi} \mathrm{d} \phi v^{3} \sin ^{2} \theta \sin \phi f_{1}^{-}\right) .
\end{aligned}
$$

The integration with respect to $v$ may be performed right away, if we use the low-temperature approximation

$$
\frac{\partial f_{0}}{\partial \epsilon} \simeq-\delta\left(\epsilon-\epsilon_{\mathrm{F}}\right)=-\frac{1}{m v_{\mathrm{F}}} \delta\left(v-v_{\mathrm{F}}\right)
$$


Transforming all integrals over $\phi$ to the interval $\left(0, \frac{1}{2} \pi\right)$ and substituting $t=r \sin \phi$, we find after rearrangement of terms

$$
\begin{aligned}
j(r)= & \frac{|e|^{2} m^{2} v^{2} \mathrm{~F}}{2 \pi^{3} h^{3} r} \int_{0}^{r} \mathrm{~d} t \frac{t^{2}}{\left[\left(r^{2}-t^{2}\right)\left(y^{2}-t^{2}\right)\right]^{\frac{1}{2}}} \int_{0}^{\pi} \mathrm{d} \theta \sin ^{2} \theta \\
& \times\left\{\int_{t}^{r} \mathrm{~d} y E(y)\left[\exp \left(-u_{+} / \sin \theta\right)+\exp \left(-u_{-} / \sin \theta\right)\right]\right. \\
& \left.+\int_{r}^{a} \mathrm{~d} y E(y)\left[\exp -u_{+} / \sin \theta+\exp \left(u_{-} / \sin \theta\right)\right]\right\},
\end{aligned}
$$

where $u_{+}=\left[\left(r^{2}-t^{2}\right)^{\frac{1}{2}} \pm\left(y^{2}-t^{2}\right)^{\frac{l}{2}}\right] / \lambda, \lambda=\tau v_{\mathrm{F}}$, the mean free path of the electrons. Following Dingle $\left.{ }^{5}\right)$ we define

$$
T_{n}(u)=\frac{1}{2} \int_{0}^{\pi} \mathrm{d} \theta \sin ^{n-1} \theta \exp (--u / \sin \theta) .
$$

For free electrons we further have the well known relations $m^{3} v_{\mathrm{F}}^{3} / \hbar^{3}=$ $k_{\mathrm{F}}^{3}=3 \pi^{2} n, \sigma_{0}=n|e|^{2} \tau / m$. So we finally arrive at the following expression for the current:

$$
\begin{aligned}
j(r)= & \frac{3 \sigma_{0}}{\pi \lambda r}\left(\int_{0}^{r} \mathrm{~d} y E(y) \int_{0}^{r} \mathrm{~d} t t^{2} \frac{T_{3}\left(u_{+}\right)+T_{3}\left(u_{-}\right)}{\left[\left(r^{2}-t^{2}\right)\left(y^{2}-t^{2}\right)\right]^{\frac{1}{2}}}\right. \\
& \left.+\int_{r}^{a} \mathrm{~d} y E(y) \int_{0}^{r} \mathrm{~d} t t^{2} \frac{T_{3}\left(u_{+}\right)+T_{3}\left(-u_{-}\right)}{\left[\left(r^{2}-t^{2}\right)\left(y^{2}-t^{2}\right)\right]^{\frac{1}{2}}}\right) .
\end{aligned}
$$

It will be convenient to rewrite (3.11) in a short-hand notation,

$$
j(r)=\sigma_{0} \int_{0}^{a} \mathrm{~d} y F(r, y) E(y),
$$

where

$$
F(r, y)=\left[\begin{array}{ll}
F_{1}(r, y) & \text { for } y \leqslant r \\
F_{2}(r, y) & \text { for } y \geqslant r
\end{array}\right.
$$

The definitions of $F_{1}$ and $F_{2}$ follow directly from (3.12). Analytical properties of the function $F(r, y)$ are given in the appendix.

4. Conclusion. In this paper we have given a theoretical description of the anomalous skin effect in a long cylinder placed in an a.c. magnetic field parallel to the cylinder axis. The current density in the sample is 
governed by the integral equation (2.8), the kernel of which is derived in section 3 . The result can be formulated explicitly as follows:

$$
F(r, y)=\left[\begin{array}{ll}
\frac{3}{\pi \lambda r} \int_{0}^{y} \mathrm{~d} t t^{2} \frac{T_{3}\left(u_{+}\right)+T_{3}\left(u_{-}\right)}{\left[\left(r^{2}-t^{2}\right)\left(y^{2}-t^{2}\right)\right]^{\frac{1}{2}}} & \text { for } y \leqslant r, \\
\frac{3}{\pi \lambda r} \int_{0}^{r} \mathrm{~d} t t^{2} \frac{T_{3}\left(u_{+}\right)+T_{3}\left(-u_{-}\right)}{\left[\left(r^{2}-t^{2}\right)\left(y^{2}-t^{2}\right)\right]^{\frac{1}{2}}} & \text { for } y \geqslant r,
\end{array}\right.
$$

where $u_{ \pm}=\left[\left(r^{2}-t^{2}\right)^{\frac{1}{2}} \pm\left(y^{2}-t^{2}\right)^{\frac{1}{2}}\right] / \lambda$. These expressions are unsuitable for analytical treatment. Numerical evaluation is in progress and will be published in a subsequent paper, where also a comparison with experimental data will be given.

\section{APPENDIX}

The function $T_{n}(u)$, defined in (3.11) can be identified with a repeated integral of the modified Bessel function ${ }^{8}$ )

$$
T_{0}(u)=K_{0}(u) ; \quad T_{n}(u)=\int_{u}^{\infty} T_{n-1}(w) \mathrm{d} w .
$$

Tabulated values of these in tegrals are given by Bickley and Nayler ${ }^{9}$ ). Series expansions for small values of the argument may be found in Dingle's paper ${ }^{5}$ ). We have, for instance,

$$
\Gamma_{3}(u) \simeq \frac{1}{4} \pi-u+\frac{1}{4} \pi u^{2} .
$$

From the definition of the functions $F_{1}(x, y)$ and $F_{2}(x, y)$ [see (3.12) and (3.13)] follows that both are singular for $x=y$. To make a numerical integration feasible, we split off the singular part by writing, for $y>x$

$$
\begin{aligned}
F_{2}(x, y)= & \frac{3}{\pi \lambda x} \int_{0}^{x} \mathrm{~d} t t^{2} \frac{\frac{1}{2} \pi}{\left[\left(x^{2}-t^{2}\right)\left(y^{2}-t^{2}\right)\right]^{\frac{1}{2}}} \\
& +\frac{3}{\pi \lambda x} \int_{0}^{x} \mathrm{~d} t t^{2} \frac{T_{3}\left(u_{+}\right)+T_{3}\left(u_{-}\right)-\frac{1}{2} \pi}{\left[\left(x^{2}-t^{2}\right)\left(y^{2}-t^{2}\right)\right]^{\frac{1}{2}}}
\end{aligned}
$$

The second integral is regular. The first one can be expressed in terms of complete elliptic integrals ${ }^{10}$ ) as follows 


$$
\begin{aligned}
\frac{3}{\pi \lambda x} & \int_{0}^{x} \mathrm{~d} t t^{2} \frac{\frac{1}{2} \pi}{\left[\left(x^{2}-t^{2}\right)\left(y^{2}-t^{2}\right)\right]^{\frac{1}{2}}} \\
= & \frac{3 y}{2 \lambda x} \int_{0}^{1} \mathrm{~d} v\left\{\left[\left(1-v^{2}\right)\left(1-m v^{2}\right)\right]^{-\frac{1}{2}}-\left(1-v^{2}\right)^{-\frac{1}{2}}\left(1-m v^{2}\right)^{\frac{1}{2}}\right\} \\
& =\frac{3 y}{2 \lambda x}\{K(m)-E(m)\}, \quad \text { with } m=x^{2} / y^{2} \leqslant 1 .
\end{aligned}
$$

The limiting behaviour for $x \simeq y$ is

$$
\frac{3}{4 \lambda}\left\{\ln \frac{8 y}{y-x}-2\right\} \text {. }
$$

So in the neighbourhood of $x=y$ we may write

$$
F_{2}(x, y)=\frac{3}{4 \lambda} \ln \frac{y}{y-x}+f_{2}(x, y)
$$

where $f_{2}(x, y)$ is a regular function. A similar analysis for $x>y$ gives

$$
F_{1}(x, y)=\frac{3}{4 \lambda} \ln \frac{y}{x-y}+f_{1}(x, y)
$$

\section{REFERENCES}

1) Pippard, A. B., Proc. Roy. Soc. A 191 (1947) 385.

2) Reuter, G. E. H. and Sondheimer, E. H., Proc. Roy. Soc A 195 (1948) 336.

3) Cotti, P., Phys. Kondens. Materie 3 (1964) 40.

4) Wyder, P., Phys. Kondens. Materie 3 (1965) 263, 292.

5) Dingle, R. B., Proc. Roy. Soc. A 201 (1950) 545.

6) Van de Klundert, L. J. M., Gijsbertse, E. A. and Van der Marel, L. C., Phys. Letters 40 A (1972) 373.

7) Fuchs, K., Proc. Camb. Phil. Soc. 34 (1938) 100.

8) Abramowitz, M. and Stegun, I. A., Handbook of Mathematical Functions, Dover Publ. (New York, 1965) ch. 11.

9) Bickley, M. G. and Nayler, J., Phil. Mag. 7 (1935) 20, 243.

10) Abramowitz, M. and Stegun, I. A., op. cit., ch. 17. 